\title{
On some fields of meromorphic functions on fibers
}

\author{
By \\ Takashi Окамо*

\section{\$1. Introduction}

1. 1. In this paper we consider the extension problem of meromorphic functions on fibers of complex analytic fiber spaces to neighborhoods of the fibers.

Let $X \stackrel{\pi}{\rightarrow} Y$ be a complex analytic fiber space, where $X$ and $Y$ are normal and connected complex spaces and $\pi$ is a proper holomorphic mapping of $X$ onto $Y$ with irreducible fibers. We denote by $K_{t}$ the meromorphic function field of a fiber $X_{t}:=\pi^{-1}(t)$, and by $K_{t}^{\prime}$ the subfield of $K_{t}$ consisting of all elements of $K_{t}$ which can be extended to some neighborhoods of $X_{t}$. By [6] or [9], the field $K_{t}$ is isomorphic to a finite algebraic extension of a rational function field.

We discuss here the following problem.

Let $f_{1}, \cdots, f_{l}$ be meromorphic functions on $X$ and $g$ be a meromorphic function on a fiber $X_{t}$ which is dependent on $f_{1, t}, \cdots f_{l, t}$, where $f_{i, t}(i=1, \cdots, l)$ is the analytic restriction of $f_{i}$ to $X_{t}$. Then, can we extend the function $g$ to a meromorphic function on some neighborhood of $X_{t}$ ?

We can answer this problem as follows.

(I) The complement of the set $\{t \in Y \mid$ any meromorphic function on $X_{t}$ which is dependent on $f_{1, t}, \cdots, f_{l, t}$ can be extended to some neighborhoods of $\left.X_{t}\right\}$ is nowhere dense in $Y$.

The proof of this theorem is essentially due to the Stein factorization of a proper holomorphic mapping. This notion (or the

Received January 30, 1967

Communicated by S. Nakano.

* Department of Engineering Mathematics, Faculty of Engineering, Nagoya University. 
notion of complex base) is useful to research dependency of holomorphic or meromorphic mappings (for example, see [5], [6], [8], [9]).

Using (I) we obtain:

(II) The set $\left\{t \in Y \mid K_{t}^{\prime}\right.$ is not algebraically closed in $\left.K_{t}\right\}$ is nowhere dense in $Y$.

Furthermore, by a similar method to the proof of (I) we have:

(III) If the transcendence degree of $K_{t}^{\prime}$ over the complex number field $\boldsymbol{C}$ is equal to the (complex) dimension of the fiber $X_{t}$, then $K_{t}^{\prime}=K_{t}$.

1. 2. In this paper, we assume all complex spaces to be reduced, and we denote the complex projective space of dimension $m$ by $\boldsymbol{P}_{m}$, and the Osgood space of dimension $l$ by $\mathbb{P}^{l}$.

We recall here the concepts of rank and of degeneracy of mappings.

Let $\sigma: M \rightarrow N$ be a holomorphic mapping of an irreducible complex space $M$ to a complex space $N$. We define the local rank of $\sigma$ at a point $x$ of $M$ by $\operatorname{dim}_{x} M-\operatorname{dim}_{x} \sigma^{-1}(\sigma(x))$ and denote it by $r_{x}(\sigma)$. Further we define the rank of $\sigma$ by $\sup _{x \equiv M} r_{x}(\sigma)$ and denote it by $r(\sigma)$.

Now, if $r_{x}(\sigma) \neq r(\sigma)$ for a point $x$ of $M$, we call this point $x a$ point of degeneracy of $\sigma$. By R. Remmert [8], the set of all points of degeneracy is an analytic subset, and any holomorphic mapping without points of degeneracy (we say such a mapping is non-degenerated or is of constant rank) to a normal complex space whose dimension is equal to the rank of the mapping is an open mapping.

\section{\$2. Some remarks on fiber spaces and meromorphic mappings}

2.1. Let $X$ and $Y$ be complex spaces and $\left\{X_{i}\right\}$ be the set of irreducible components of $X$.

Now let $f$ be a correspondence between $X$ and $Y$. We denote the graph of $f$ by $G$ and the natural projections of $G$ to $X$ and $Y$ by $\breve{f}$ and $\hat{f}$ respectively. Conforming to [9], we call the correspondence $f$ to be a meromorphic mapping of $X$ to $Y$ if the following condi- 
tions are satisfied;

(a) there is a dense open set of $X$ on which $f$ defines a holomorphic mapping to $Y$,

(b) the graph $G$ is an analytic subset of $X \times Y$, and $f^{-1}\left(X_{i}\right)$ is an irreducible component of $G$ for each $X_{i}$,

(c) the projection $\breve{f}$ is proper.

Let $f$ be a meromorphic mapping of $X$ to $Y$. We call a point $x$ of $X$ a singular point of $f$ if $f$ is not holomorphic at $x$, and call $f$ to be proper (resp. surjective) if $\hat{f}$ is proper (resp. surjective). Further we define the rank of $f$ by $r(\hat{f})$ and denote it by $r(f)$. Moreover we say that a meromorphic mapping $f$ of $X$ to $Y$ is bimeromorphic if the correspondence $f$ defines a meromorphic mapping of $Y$ to $X$.

Next, we recall some fundamental properties of meromorphic mappings.

(i) The set of all singular points of a meromorphic mapping is an analytic subset.

(ii) A meromorphic mapping of a certain complex space $X$ to the complex projective space $\boldsymbol{P}^{1}$ which maps $X$ not constantly to $\infty$ is nothing but a meromorphic function in the usual sense.

(iii) Let $X, Y$ and $Z$ be complex spaces and $f$ and $g$ be meromorphic mappings of $X$ to $Y$ and of $Y$ to $Z$ respectively. We define naturally a correspondence between $X$ and $Z$ such that a point $x$ of $X$ corresponds to the subset $g(f(x))$ of $Z$. If there is a dense open set $U$ of $X$ on which the above correspondence between $X$ and $Z$ is single-valued, then we can define naturally one meromorphic mapping $h$ of $X$ to $Z$ such that $h(x)=g(f(x))$ for $x \in U$. We denote it by $g \circ f$. In particular, if $X$ is a subspace of $Y$ and $f$ is the inclusion map, we denote $g \circ f$ by $g \| X$.

(iv) Let $X, Y_{1}, \cdots, Y_{l}$ be complex spaces and $f_{i}$ be a meromorphic mapping of $X$ to $Y_{i}(i=1, \cdots, l)$. Then we can naturally define one meromorphic mapping of $X$ to the product space $Y_{1} \times \cdots \times Y_{l}$. We denote it by $f_{1} \times \cdots \times f_{l}$.

(v) Let $X$ and $Y$ be irreducible complex spaces of the same 
dimension and $f$ be a proper and surjective meromorphic mapping of $X$ to $Y$. Then there is a thin analytic subset $N$ of $Y$ such that $f$ is holomorphic on $X-\check{f}\left(\hat{f}^{-1}(N)\right)$ and the map $f \|\left(X-\breve{f}\left(\hat{f}^{-1}(N)\right)\right.$ is a proper holomorphic covering map of $X-\breve{f}\left(\hat{f}^{-1}(N)\right)$ to $Y-N$. We call such a meromorphic mapping to be a meromorphic covering.

Next, we recall the notion of dependency of meromorphic mappings.

Let $X, Y$ and $Z$ be complex spaces and $f$ and $g$ be meromorphic mappings of $X$ to $Y$ and of $X$ to $Z$ respectively. Then we say that $g$ depends on $f$ if $r(f \times g)=r(f)$. Further let $f_{1}, \cdots, f_{l}$ be meromorphic functions on $X$. Then we say that the system $\left\{f_{1}, \cdots, f_{l}\right\}$ is independent if $r\left(f_{1} \times \cdots \times f_{l}\right)=l$.

2.2. Let $X$ and $Y$ be complex spaces and $\pi$ be a proper holomorphic mapping of $X$ to $Y$. We denote the set of all connected components of all fibers of the map $\pi$ by $X^{\prime}$. By [1] we can define on the set $X^{\prime}$ a topology and a complex structure which have the following properties;

(a) the natural maps $\pi_{1}: X \rightarrow X^{\prime}$ and $\pi_{2}: X^{\prime} \rightarrow Y$ are holomorphic.

(b) an arbitrary map $h$ of $X^{\prime}$ to a complex space $Z$ such that $h \circ \pi_{1}$ is holomorphic is holomorphic.

We call this sequence $X \stackrel{\pi_{1}}{\rightarrow} X^{\prime} \stackrel{\pi_{2}}{\rightarrow} Y$ the Stein factorization of $\pi$.

Proposition 1. Let $X$ be a compact irreducible complex space, and $f_{1}, \cdots, f_{l}$ be meromorphic functions on $X$. We put $F=f_{1} \times \cdots$ $\times f_{l}, G=$ the graph of $F$. Let $\widetilde{G} \stackrel{\mu}{\rightarrow} G$ be the normalization of $G$ and $\widetilde{G} \stackrel{h_{1}}{\rightarrow} H \stackrel{h_{2}}{\rightarrow} \mathbb{P}^{l}$ be the Stein factorization of the proper holomorphic mapping $\hat{F} \circ \mu$, where $\hat{F}$ is the natural projection of $G$ to $\boldsymbol{P}^{l}$.

Then, for any meromorphic function $g$ on $X$ dependent on $F$, there is a meromorphic function $g^{\prime}$ on $H$ such that $g=g^{\prime} \circ h_{1} \circ \mu^{-1} \circ \check{F}^{-1}$.

Proof. Since $X$ and $\widetilde{G}$ are bimeromorphically equivalent, we may assume that $X$ is normal and connected and $F$ is holomorphic on $X$. Under these assumptions we may identify the sequence $\widetilde{G} \stackrel{h_{1}}{\rightarrow} H \stackrel{h_{2}}{\rightarrow} \boldsymbol{P}^{l}$ with the Stein factorization $X \stackrel{h_{1}}{\rightarrow} X^{\prime} \stackrel{h_{2}}{\rightarrow} \mathbb{P}^{l}$ of the proper holomorphic mapping $F$. 
Let $S(g)$ be the singular set of $g$. Since $X$ is compact and $g$ depends on $F$, there is a polynomial $P_{s}\left(X_{1}, \cdots, X_{l}\right) X^{s}+\cdots+P_{0}$ $\left(X_{1}, \cdots, X_{l}\right)$, where $s>0$, such that $P_{s}\left(f_{1}, \cdots, f_{l}\right) g^{s}+\cdots+P_{0}\left(f_{1}, \cdots, f_{l}\right)$ $\equiv 0$ on $X$ and $P_{s}\left(f_{1}, \cdots, f_{l}\right) \neq 0$ on $X$ (see [9], p. 864). Now we take a point $z=\left(z_{1}, \cdots, z_{l}\right)$ of $F(X)$ such that $z_{i} \neq \infty$ for all $i$ and $P_{s}\left(z_{1}, \cdots, z_{l}\right) \neq 0$. Let $x$ be a point of $F^{-1}(z)$. Then $g(x)$ is a finite set in $\boldsymbol{P}^{1}$ since $P_{s}\left(z_{1}, \cdots, z_{l}\right) \neq 0$. This fact and the normality of $X$ yield the holomorphy of $g$ at $x$ (see [9], Prop. 3. 1. 3). Hence $F(S(g)) \neq F(X)$.

Since $F(X)$ is an irreducible complex space, $F(S(g))$ is a thin analytic subset of $F(X)$ and so $F^{-1}(F(S(g)))$ is a thin analytic set of $X$. We put $X_{0}=X-F^{-1}(F(E) \cup F(S(g)))$, where $E$ is the set of degeneracy of $F . \quad\left(F^{-1}(F(E))\right.$ is thin in $X$.) We denote the Stein factorization of the proper holomorphic mapping $F \mid X_{0}$ by $X_{0} \rightarrow X_{0}^{\prime} \rightarrow$ $F\left(X_{0}\right)$. Then we may consider that $X_{0}^{\prime}=h_{1}\left(X_{0}\right)\left(\subset X^{\prime}\right)$. For a point $x$ of $X_{0}, r_{x}(F)=r_{x}(F \times g)$ because $r_{x}(F) \leq r_{x}(F \times g) \leq r(F \times g)=r(F)$ $=r_{x}(F)$. Hence $g$ is constant along each connected component of $F^{-1}(z)$ for any $z$ of $F\left(X_{0}\right)$ (see [8], p. 300). Therefore we obtain a holomorphic function $g_{0}^{\prime}$ on $X_{0}^{\prime}$ such that $g \| X_{0}=g_{0}^{\prime} \circ h_{1}$.

Put $G(g)=$ the graph of $g$, and $G^{\prime}=$ the Image of $G(g)$ by the map $h_{1} \times 1$ of $X \times \boldsymbol{P}^{1}$ to $X^{\prime} \times \mathbb{P}^{1}$. Then $G^{\prime}$ gives a moromorphic function $g^{\prime}$ on $X^{\prime}$ such that $g^{\prime} \| X_{0}^{\prime}=g_{0}^{\prime}$.

Remark. Proposition 1 can be generalized as follows:

Let $\pi: X \rightarrow Y$ be a proper holomorphic mapping, where $X$ is irreducible, and $f_{1}, \cdots, f_{l}$ be meromorphic functions on $X$. We put $\sigma=f_{1} \times \cdots \times f_{l} \times \pi, G=$ the graph of $\sigma$, and $\hat{\sigma}, \tilde{\sigma}=$ the natural projections of $G$ to $\mathbb{P}^{l} \times Y$ and to $X$. Let $\widetilde{G}^{\mu} \rightarrow G$ be the normalization of $G$ and $\widetilde{G} \stackrel{h_{1}}{\rightarrow} H \stackrel{h_{2}}{\rightarrow} \mathbb{P}^{l} \times Y$ be the Stein factorization of $\hat{\sigma} \circ \mu$.

Then, for any meromorphic function $g$ on $X$ dependent on $\sigma$, there is a meromorphic function $g^{\prime}$ on $H$ such that $g=g^{\prime} \circ h_{1} \circ \mu^{-1} \circ \check{\sigma}^{-1}$.

Proposition 2. Let $V$ be an irreducible analytic subspace of $\boldsymbol{P}_{m}$. Then any element of the field $K(V)$ of all meromorphic functions on $V$ is the restriction of a rational function of $\boldsymbol{P}_{m}$. 
Furthermore let $\left\{f_{1}, \cdots, f_{l}\right\}$ be a transcendence base of $K(V)$ over the complex number field $\mathbb{C}$. Then the degree of $K(V)$ over the field $\mathbb{C}\left(f_{1}, \cdots, f_{l}\right)$ is equal to the number of sheet of the meromor. phic covering map $F: V \rightarrow \mathbb{P}^{l}$, where $F=f_{1} \times \cdots \times f_{l}$.

Proof. Let $K_{R}(V)$ be the subfield of $K(V)$ consisting of all elements of $K(V)$ which can be extended to a rational function of $\boldsymbol{P}_{m}$. Then the transcendence degrees of $K(V)$ and $K_{R}(V)$ over $C$ are equal to the dimension of $V$. Let $\left\{f_{1}, \cdots, f_{l}\right\}$ be a transcendence base of $K_{R}(V)$ over $\mathbb{C}$ and $F$ be the meromorphic mapping $f_{1} \times \cdots \times f_{l}$ of $V$ to $\mathbb{P}^{l}$. Then $F$ is a meromorphic covering map, because $\operatorname{dim} V=l$ $=\operatorname{dim} \mathbb{P}^{l}$ and the system $\left\{f_{1}, \cdots, f_{l}\right\}$ is independent. So there is an analytic subset $N$ of $\mathbb{P}^{l}$ such that $F$ is holomorphic on $V-F^{-1}(N)$ and $F \|\left(V-F^{-1}(N)\right)$ is a proper unramified holomorphic covering map to $\mathbb{P}^{l}-N$. We put $b=$ the number of sheet of $F \|\left(V-F^{-1}(N)\right)$, $d=\left[K(V): \mathbb{C}\left(f_{1}, \cdots, f_{l}\right)\right]$ and $d^{\prime}=\left[K_{R}(V): \mathbb{C}\left(f_{1}, \cdots, f_{l}\right)\right]$. Then clearly $b \geq d \geq d^{\prime}$, because any element $f$ of $K(V)$ satisfies; $f^{b}+$ $H_{b-1} f^{b-1}+\cdots+H_{0}=0$, where $H_{i}(i=0,1, \cdots, b-1)$ is a suitable rational function of $\mathbb{P}^{l}$ which is considered as an element of $\mathbb{C}\left(f_{1}, \cdots, f_{l}\right)$.

On the other hand, we can find an element $g$ of $K_{R}(V)$ whose degree over $\mathbb{C}\left(f_{1}, \cdots f_{l}\right)$ is not smaller than $b$. In fact, fix a point $p$ of $\mathbb{P}^{l}-N$, and put $F^{-1}(p)=\left\{p_{1}, \cdots, p_{b}\right\}$. Then we can easily find two linear forms $w_{1}=a_{0} z_{0}+\cdots+a_{n} z_{m}, w_{2}=b_{0} z_{0}+\cdots+b_{m} z_{m}$, for a system of homogeneous coordinate $\left\{z_{c}, \cdots, z_{m}\right\}$ of $\mathbb{P}_{m}$, such that $w_{1}\left(p_{i}\right) \neq 0$ for all $i$, and $\frac{w_{2}\left(p_{1}\right)}{w_{1}\left(p_{i}\right)} \neq \frac{w_{2}\left(p_{1}\right)}{w_{1}\left(p_{1}\right)}$ (for $\left.i \neq j\right)$. Now we put $\tilde{\alpha}=\frac{w_{2}}{w_{1}}$ and $\alpha=\tilde{\alpha} \| V$. Then it can be easily proved that the degree of $\alpha$ over $\mathbb{C}\left(f, \cdots, f_{l}\right)$ is not smaller than $b$.

Theorem. (H. Grauert and R. Remmert, [2], [4]). Let $X$ and $Y$ be complex spaces and $\sigma$ be a proper holomorphic mapping of $X$ to the product space $\mathbb{P}_{m} \times Y$ with discrete fibers. Let $U$ be a relatively compact Stein open set of $Y$. We put $X_{U}=\sigma^{-1}\left(\mathbb{P}_{m} \times U\right)$.

Then, there is a natural number $N$ and a biholomorphic mapping $\omega$ of $X_{U}$ to an analytic subspace of the product space 
$\boldsymbol{P}_{m} \times U \times \boldsymbol{P}_{N}$ such that $\sigma \mid X_{U}=p \circ \omega$, where $p$ is the natural projection of $\boldsymbol{P}_{m} \times Y \times \boldsymbol{P}_{N}$ to $\boldsymbol{P}_{m} \times Y$.

Proposition 3. Let $\pi: X \rightarrow Y$ be a proper holomorphic mapping of a normal complex space $X$ onto a complex space $Y$. Then the set $\left\{t \in Y \mid\right.$ the space $\pi^{-1}(t)$ is not locally irreducible $\}$ is nowhere dense in $Y$.

The proof of this proposition is essentially due to W. Thimm [11]. We prove this in the next section.

\section{§3. Proof of Proposition 3}

To prove our proposition we use local descriptions of the normal complex space $X$. Therefore we start by setting the following notations. We put;

$$
\begin{aligned}
& T=\left\{\left(t_{1}, \cdots, t_{n}\right) \in C^{n}|\quad| t_{i} \mid<\tau_{i} ; i=1, \cdots, n\right\}, \\
& \left.Z_{m}=\left\{z_{1}, \cdots, z_{m}\right) \in \mathbb{C}^{m}|\quad| z_{j} \mid<\zeta_{j} ; j=1, \cdots, m\right\}, \\
& D_{m}=T \times Z_{m}, p=\text { the natural projection of } D_{m} \text { to } T, \\
& Z_{m, t}=p^{-1}(t), \text { where } t \text { is a point of } T .
\end{aligned}
$$

Now let $A$ be an analytic set of $D_{m}$ and $T_{0}$ be the set $\{t \in T \mid$ $\left.Z_{m, t} \cap A=Z_{m, t}\right\}$. We consider the following condition $(*)$ for a point $x$ of $D_{m}$ with respect to $A$ :

(*) The point $p(x)$ does not belong to $T_{\mathrm{c}}$ and there is a fundamental system of neighborhoods $\left\{U_{i}\right\}$ of the point $x$ which satisfies the following condition $(C)$;

(C) for a curve $C$ in $U_{i}$ such that $C \cap A=\phi$ and $p(C(0))=$ $p(C(1))=p(x)$, there is a deformation of the curve $C$ to a curve in $U_{i} \cap Z_{m, p(x)}$ through the space $U_{i}-A$, with the end points $C(0)$ and $C(1)$ fixed.

Lemma 3.1. Let $M$ be a connected normal complex space and $r$ be a proper holomorphic covering map of $M$ to $D_{m}$ which is unramified over $D_{m}-A$. If, for a point $x$ of $M$, the point $r(x)$ 
satisfies the condition (*) with respect to $A$, then $x$ is an irreducible point of the fiber $(p \circ r)^{-1}((p \circ r)(x))$.

Proof. Suppose that $r(x)$ satisfies the condition $(*)$ with respect to $A$. We put $M_{x}=(p \circ r)^{-1}(p \circ r)(x)$. Then $M_{x} \cap r^{-1}(A)$ is a thin analytic set of $M_{x}$, and $M_{x}-r^{-1}(A)$ is non-singular. Hence $x$ is an irreducible point of $M_{x}$ if and only if there is a fundamental system of neighborhoods $\left\{U_{k}^{\prime}\right\}$ of the point $x$ in the space $M_{x}$ such that $U_{k}^{\prime}-r^{-1}(A)$ is connected.

Take a connected neighborhood $V$ of $x$ in the space $M$ such that $V \cap M_{x}$ is sufficiently small and,

(a) the open set $r(V)$ satisfies the condition (C) with respect to $A$ at $r(x)$,

(b) the mapping $r \mid V: V \rightarrow r(V)$ is proper.

We put $U^{\prime}=V \cap M_{x}$. Then from the above (a) and (b) $U^{\prime}-r^{-1}(A)$ is connected. In fact, let $x_{1}$ and $x_{2}$ be points of $U^{\prime}-r^{-1}(A)$. Since $V$ is connected and normal, we can connect $x_{1}$ to $x_{2}$ by a curve $\widetilde{C}$ in $V-r^{-1}(A)$. We put $C=r(\widetilde{C})$. By (a), $C$ can be deformed to a curve in $Z_{m, p(r(x))} \cap(r(V)-A)$ through the space $r(V)-A$, fixing the end points. On the other hand, the map $r \mid V$ is a proper unramified covering over $r(V)-A$. Hence we can deform $\widetilde{C}$ to a curve of $U^{\prime}-r^{-1}(A)$ through the space $V-r^{-1}(A)$, by lifting the deformation of the curve $C$. Hence $U^{\prime}-r^{-1}(A)$ is connected.

Lemma 3.2. We put; $Z_{m-1}=\left\{\left(z_{1}, \cdots, z_{m-1}\right) \in C^{m-1}|| z_{j} \mid<\zeta_{j} ; j=1\right.$, $\cdots, m-1\}$, and $D_{m-1}=T \times Z_{m-1}$ and $q=$ the natural projection of $D_{m}$ to $D_{m-1}$.

Suppose that $q \mid A$ is a proper holomorphic covering map onto $D_{m-1}$ and it is unramified over $D_{m-1}-B$, where $B$ is a thin analytic set of $D_{n-1}$.

Then, for a point $x$ of $D_{m}$, if $q(x)$ satisfies the condition (*) with respect to $B$ then $x$ also satisfies the condition (*) with respect to $A$. 
Proof. Let $W$ be a neighborhood of $x$. Then we can find a neighborhood $U$ of $x$ having the following properties;

(a) $U \subset W$,

(b) $U$ is of the form $q(U) \times D$, where $D$ is a disk of $\mathbb{C}^{1}$,

(c) $q(U)$ satisfies the condition $(C)$ at $q(x)$ with respect to $B$, and

(d) $q \mid A \cap U: A \cap U \rightarrow q(U)$ is proper.

Then we can prove that the open set $U$ satisfles the condition (C) at $x$ with respect to $A$ by the same methods as in [11]. We give only an outline of the proof.

Let $C$ be a curve in $U-A$ with the end points $C(0)$ and $C(1)$ such that $p(C(0))=p(C(1))=p(x)$. Without loss of generality, we may assume that $q(C(0))$ and $q(C(1))$ do not belong to $B$, because $Z_{m-1, p(x)} \cap B \neq Z_{m-1, p(x)}$ by above $(c)$ and so we can replace the end points by two suitable points in $U \cap Z_{m, p(x)}-\left(A \cup q^{-1}(B)\right)$ which are connected to $C(0)$ and $C(1)$ by arcs in $U \cap Z_{m, p(x)}-A$ respectively. Moreover we may assume that $q(C)$ is disjoint with $B$, because the curve $C$ can be deformed, fixing the end points, to a curve which is sufficiently near to $C$ and whose projection to $q(U)$ is disjoint with $B$ (see $[11], \S 2)$. Under these assumptions, $q(C)$ can be deformed by the above property (c) to a curve of $q(U) \cap q\left(Z_{m, p(x)}\right)$ though the space $q(U)-B$ with the end points fixed. On the other hand, since $q \mid A$ is proper and unramified over $D_{m-1}-B$, we can construct a deformation of $C$ in $U-A$ with the desired properties lying above the deformation of $q(C)$ (see [10], 2 and [11], §2).

Lemma 3.3. We suppose that $A$ is purely 1-codimentional in $D_{m}$, and put $D_{m}^{*}=\left\{x \in D_{m} \mid x\right.$ satisfies the condition (*) with respect to $A\}$.

Then $p\left(K_{m}-D_{m}^{*}\right)$ is nowhere dense in $T$ for any relatively compact subset $K_{m}$ of $D_{m}$.

Proof. We prove the lemma by induction on $m$. If $m=0$, it is 
trivial. So we suppose that $m>0$ and that the result holds for $m-1$.

We denote the $\varepsilon$-neighborhood of the set $T_{0}$ by $T_{0}(\varepsilon)$. Then $p\left(K_{m}-D_{m}^{*}\right)$ is nowhere dense in $T$ if and only if it is nowhere dense in $T-T_{0}(\varepsilon)$ for any positive number $\varepsilon$. We put $K_{m}(\varepsilon)=K_{m}-p^{-1}\left(T_{0}(\varepsilon)\right)$.

Now let $x$ be a point of $D_{m}$. If $x \notin A$, take a neighborhood $U_{m}(x)$ of $x$ such that $U_{m}(x) \cap A=\phi$. Then any point of $U_{m}(x)$ satisfies the condition (*) with respect to $A$. Next we suppose $x \in A-p^{-1}\left(T_{0}\right)$. Then, since $A$ is purely codimensional 1 , we can find a neighborhood $V_{m}(x)$ of $x$ satisfying the following properties:

(a) $V_{m}(x)$ is the product of two polycylinders $T(x)$ and $Y_{m}(x)$, where $T(x)$ and $Y_{m}(x)$ are defined as follows;

$$
\begin{aligned}
& T(x)=\left\{\left(t_{1}^{\prime}, \cdots, t_{n}^{\prime}\right) \in C^{n}|\quad| t_{i} \mid<\tau_{i}^{\prime} ; i=1, \cdots, n\right\}, \\
& Y_{m}(x)=\left\{\left(y_{1}, \cdots, y_{m}\right) \in C^{m}|\quad| y_{j} \mid<\eta_{j} ; j=1, \cdots, m\right\},
\end{aligned}
$$

where $t_{i}^{\prime}=t_{i}-t_{i}(p(x))$ and $y_{j}=\sum_{k=1}^{m} c_{j k} z_{k}+d_{j}$ such that $y_{j}(x)=0$ (for any $j$ ) and the matrix $\left(c_{j k}\right)$ is non-singular, and $\tau_{\imath}^{\prime}$ and $\eta_{j}$ are suitable positive numbers.

(b) Let $Y_{m-1}(x)=\left\{\left(y_{1}, \cdots, y_{m-1}\right) \in C^{m-1}|| y_{j} \mid<\eta_{j} ; j=1, \cdots, m-1\right\}$ and $V_{m-1}(x)=T(x) \times Y_{m-1}(x)$ and $q=$ the natural projection of $V_{m}(x)$ to $V_{m-1}(x)$. In this situation, $q \mid V_{m}(x) \cap A$ is a proper covering map and unramified over $V_{m-1}(x)-B$, where $B$ is an analytic subset of $V_{m-1}(x)$ purely of codimension 1 .

We denote the natural projection of $V_{m-1}(x)$ to $T(x)$ by $p_{m-1}$, and the set $\left\{s \in V_{m-1}(x) \mid s\right.$ satisfies the condition (*) with respect to $B\}$ by $V_{m-1}^{*}(x)$. Let now $U_{m-1}(x)$ be an arbitrarily fixed relatively compact open neighborhood of $q(x)$ in $V_{m-1}(x)$. Then, by the hypothesis of induction, $p_{m-1}\left(U_{m-1}(x)-V_{m-1}^{*}(x)\right)$ is nowhere dense in $T(x)$. Hence, by Lemma 3. 2, $p\left(U_{m}(x)-D_{m}^{*}\right)$ is nowhere dense in $T(x)$, where $U_{m}(x)$ is the set $q^{-1}\left(U_{m-1}(x)\right)$.

For each point $x$ of $K_{m}(\varepsilon)$ we take such an open neighborhood $U_{m}(x)$ mentioned above. Since $K_{m}(\varepsilon)$ is compact, it is covered by a finite system of such neighborhoods $U_{m}\left(x_{k}\right)$ and hence $p\left(K_{m}(\varepsilon)-D_{m}^{*}\right)$ 
is nowhere dense in $T$.

Proof of Proposition 3. We may assume that $Y$ is non-singular and $\pi$ is of constant rank. For, our assertion is of local character about $Y$ and the $\pi$-image of the set of degeneracy of $\pi$ is a thin analytic set in $Y$. Moreover we may assume; $Y=\left\{\left(t_{1}, \cdots, t_{n}\right) \in \mathbb{C}^{n} \mid\right.$ $\left.\left|t_{i}\right|<\tau_{i} ; i=1, \cdots, n\right\}$. Then, for each point $x$ of $X$, we can find a connected open neighborhood $U(x)$ such that there is a proper holomorphic covering map $r$ of $U(x)$ to $D_{m}$, where $D_{m}$ is a polycylinder which is obtained by replacing $t_{i}$ by $t_{\imath}-t_{\imath}(\pi(x))$ in $D_{m}$ of the beginning of this section.

Let $A$ be a purely one codimensional analytic set in $D_{m}$ such that $r$ is unramified over $D_{m}-A$. Further let $W$ be a relatively compact open set of $D_{m}$ containing $r(x)$ and $V(x)$ be the open set $r^{-1}(W) \cap$ $U(x)$. Then, by Lemma 3. 3, $p\left(W-D_{m}^{*}\right)$ is nowhere dense in $T(\subset Y)$ and hence $X_{t} \cap V(x)$ is locally irreducible by Lemma 3. 1 for any point $t$ of $p(W)-p\left(W-D_{m}^{*}\right)$.

For each point $x$ of $X$, we take such a neighborhood $V(x)$. Let $Q$ be a relatively compact open set of $Y$. Then the set $\pi^{-1}(\bar{Q})$ is compact and so it is covered by a finite system of open sets $V\left(x_{k}\right)$. Hence the set $\left\{t \in Y \mid X_{t}\right.$ is not locally irreducible $\}$ is nowhere dense in $Y$.

\section{§4. Meromorphic function fields on fibers}

In this section, we consider a fiber space $X \stackrel{\pi}{\rightarrow} Y$, where $X$ and $Y$ are complex spaces and $\pi$ is a proper surjective holomorphic mapping. We put $\operatorname{dim} Y=n$ and $\operatorname{dim} X=m+n$. Furthermore we assume;

(a) $X$ and $Y$ are normal and connected,

(b) $\pi$ is of constant rank, $n$,

(c) for every $t \in Y$, the fiber $X_{t}$ is irreducible.

These assumptions imply,

(d) $\pi^{-1}(U)$ is connected for any connected open set $U$ of $Y$.

From now on, we use occasionally a notation $h_{t}$ instead of $h \| X_{t}$, 
where $h$ is a meromorphic mapping of $X$ to a certain complex space and $t$ is a point of $Y$ such that $h \| X_{t}$ is defined.

Lemma 4. 1. Let $f_{1}, \cdots, f_{l}$ be meromorphic functions on $X$. We put $F=f_{1} \times \cdots \times f_{l}$ and $S(F)=$ the singular set of $F$. Then the set $\left\{t \in Y \mid X_{t} \leftarrow S(F)\right\}$ is a dense open subset of $Y$.

Let $t$ be a point of $Y$ such that $X_{t} \leftarrow S(F)$. We suppose that $\left\{f_{1, t}, \cdots, f_{l, t}\right\}$ is independent. Then there is an open neighborhood $U$ of $t$ such that $f_{i, t}(i=1, \cdots, l)$ is defined and $\left\{f_{1, t^{\prime}}, \cdots, f_{l, t^{\prime}}\right\}$ is independent for any $t^{\prime}$ of $U$. (In this case, $r(F \times \pi)=n+l$ and $\left.(F \times \pi)(X)=\mathbb{P}^{l} \times Y\right)$.

Proof. The first assertion is trivial.

Suppose that $\left\{f_{1, t}, \cdots, f_{l, t}\right\}$ is independent. We can find a point $x$ of $X_{t}$ such that $x \notin S(F)$ and $r_{x}\left(F_{t}\right)=r\left(F_{t}\right)=l$ (here we consider $F$ as a holomorphic mapping on a neighborhood of $x)$. Then $r_{x}(F \times \pi)$ $=r(F \times \pi)=n+l$ because $\operatorname{dim}_{x}(F \times \pi)^{-1}((F \times \pi)(x))=\operatorname{dim}_{x} F_{t}^{-1}\left(F_{t}(x)\right)$ $=m-r_{x}\left(F_{t}\right)=m-l$, and so $(F \times \pi)(X)=\mathbb{P}^{l} \times Y$.

Take a neighborhood $Q$ of $x$ such that $Q \cap S(F)=\phi$ and $r_{x^{\prime}}(F \times \pi)$ $=n+l$ for any point $x^{\prime}$ of $Q$. Put $U=\pi(Q)$. Since $\pi$ is of constant rank, $U$ is an open set and clearly has our desired properties.

Theorem $\mathbb{I}$. Let $t_{c}$ be a point of $Y$ and $f_{1}, \cdots, f_{l}$ be meromorphic functions on $X$ such that $f_{i, t_{0}}$ is defined for any $i$ and the system $\left\{f_{1, t_{0}}, \cdots, f_{l, t_{0}}\right\}$ is independent. We put $F=f_{1} \times \cdots \cdots \times f_{l}$, $\sigma=F \times \pi, G=$ the graph of $\sigma$, and $G_{t_{0}}=$ the graph of $F_{t_{0}}$, and we denote the normalization of $G$ by $\widetilde{G}^{\stackrel{\mu}{\rightarrow}} G$.

We suppose that;

(I) the complex space $\widetilde{G} \mid X_{t_{0}}$ (=the restriction of $\widetilde{G}$ over $X_{t_{0}}$ ) is locally irreducible.

Then there is an open neighborhood $U$ of $t_{0}$ such that any meromorphic function defined on $X_{t_{0}}$ which is dependent on $F_{t_{0}}$ can be extended to a meromorphic function on $\pi^{-1}(U)$.

Proof. Since $X$ is normal, every fiber of the map $\widetilde{G} \rightarrow X$ is connected, and $X_{t}$ is irreducible by the assumption. Hence $\widetilde{G} \mid X_{t}$ is con- 
nected for any $t$ of $Y$. On the other hand, $G \mid X_{t_{0}}$ is locally irreducible by the assumption (I). Therefore $\widetilde{G} \mid X_{t_{0}}$ is irreducible, and so $G \mid X_{t_{0}}$ is also irreducible and hence $G \mid X_{t_{0}}=G_{t_{0}}$. By these facts the space $\widetilde{G} \mid X_{t_{0}}$ is homeomorphic and bimeromorphic to the normalization $\widetilde{G}_{t_{0}}$ of $G_{t_{0}}$.

Let $\widetilde{G} \stackrel{h_{1}}{\rightarrow} H^{h_{2}} \rightarrow \boldsymbol{P}^{l} \times Y$ be the Stein factorization of the proper holomorphic mapping $\hat{\sigma} \circ \mu$, where $\hat{\sigma}$ is the natural projection of $G$ to $\boldsymbol{P}^{l} \times Y$, and $\widetilde{G}_{t_{0}}{ }_{h_{1}, t_{0}}^{\rightarrow} H_{t_{0}} \rightarrow{ }_{L_{2}, t_{0}}^{\rightarrow} \mathbb{P}^{l}$ be the Stein factorization $\hat{F}_{t_{0}} \circ \mu_{t_{0}}$, where $\mu_{t_{0}}$ is the normalization map $\widetilde{G}_{t_{0}}{ }_{t_{0}} \rightarrow G_{t_{0}}$ and $\hat{F}_{t_{0}}$ is the natural projection of $G_{t_{0}}$ to $\mathbb{P}^{l}$. From above, $h_{2}^{-1}\left(\boldsymbol{P}^{l} \times t_{0}\right)$ is also naturally homeomorphic and bimeromorpic to $H_{t_{0}}$, so we may identify $H_{t_{0}}$ with $h_{2}^{-1}\left(\boldsymbol{P}^{l} \times t_{0}\right)$.

By proposition 1, we can find a meromorphic function $g^{\prime}$ on $H_{t_{0}}$ such that $g=g^{\prime} \circ h_{1, t_{0}} \circ \mu_{0}^{-1} \circ \breve{F}_{t_{0}}^{-1}$, where $\breve{F}_{t_{0}}$ is the natural projection of $G_{t_{0}}$ to $X_{t_{0}}$.

On the other hand, the map $h_{2}: H \rightarrow P^{l} \times Y$ is proper (and surjective) with discrete fibers. Hence, by Theorem of $\$ 2$, there is a neighborhood $U$ of $t_{0}$ and a biholomorphic mapping $\omega$ of $h_{2}^{-1}\left(\boldsymbol{P}^{l} \times U\right)$ to an analytic subspace $L_{U}$ of $\mathbb{P}^{l} \times U \times \boldsymbol{P}_{N}$.

We put $g^{\prime \prime}=g^{\prime} \circ\left(\omega \| H_{t_{0}}\right)^{-1}$ on $L_{t_{0}}$. By proposition 2, there is a rational function $g^{\prime \prime \prime}$ on $\boldsymbol{P}^{l} \times \boldsymbol{P}_{N}\left(\cong \boldsymbol{P}^{l} \times t \times \boldsymbol{P}_{N}\right)$ such that $g^{\prime \prime \prime} \| L_{t_{0}}=g^{\prime \prime}$. Further we put $\widetilde{g}^{\prime \prime \prime}=g^{\prime \prime \prime} \circ \tau$, where $\tau$ is the natural projection of $\boldsymbol{P}^{l} \times U$ $\times \boldsymbol{P}_{N}$ to $\boldsymbol{P}^{l} \times \mathbb{P}_{N}$, and put $\widetilde{g}^{\prime \prime}=\widetilde{g}^{\prime \prime \prime} \| L_{U}$. Finally we set $\tilde{g}=\widetilde{g}^{\prime \prime} \circ \omega \circ h_{1} \circ$ $\mu^{-1} \circ \check{\sigma}^{-1}$ on $\pi^{-1}(U)$. Then $\widetilde{g}$ is a meromorphic function with $g=\widetilde{g} \| X_{t_{0}}$.

Remark. By the construction of $\widetilde{g}$, it is easily shown that there is a polynomial $P(t)\left(X_{0}, X_{1}, \cdots, X_{l}\right)$ with holomorphic functions on $U$ as coefficients (if necessary, replace $U$ with a smaller neighbor. hood of $\left.t_{0}\right)$ such that $P(i)\left(g, f_{1}, \cdots, f_{l}\right) \equiv 0$ on $\pi^{-1}(U)$ and $P\left(t_{0}\right)\left(X_{0}, X_{1}, \cdots, X_{l}\right) \neq 0$.

We use the following notations:

$K_{t}=$ the field of all meromorphic functions on the fiber $X_{t}$, $K_{t^{\prime}}^{\prime}=$ the subfield of $K_{t}$ consisting of all elements of $K_{t}$, which 
can be extended to neighborhoods of $Y_{t}$, $Y(k)=\{t \in Y \mid$ there is a neighborhood $U$ of $t$ in $Y$ such that the transcendence degree of $K_{t_{1}}^{\prime}=k$ for any $t_{1}$ of $\left.U\right\}$, and $Y^{\prime}=Y(0) \cup Y(1) \cup \cdots \cup Y(m)$.

Corollary. Let $f_{1}, \cdots, f_{l}$ be meromorphic functions on $X$ such that $f_{i, t}$ is defined for any $i$ and the system $\left\{f_{1, t}, \cdots, f_{l, t}\right\}$ is independent for any $t$ of $Y$. We set $K_{t}^{\prime \prime}(f)=$ the algebraic closure of the field $C\left(f_{1, t}, \cdots, f_{l, t}\right)$ in $K_{t}$. Then the set $\left\{t \in Y \mid K_{t}^{\prime \prime}(f) \mp K_{t}^{\prime}\right\}$ is nowhere dense in $Y$.

Proof. By Proposition 3 there is a nowhere dense set $Y_{0}$ of $Y$ such that any point of $Y-Y_{0}$ satisfies the condition (I) of Theorem I. Hence our assertion is proved by Theorem I.

Theorem II. The set $Y_{1}=\left\{t \in Y \mid K_{t}^{\prime}\right.$ is not algebraically closed in $\left.K_{t}\right\}$ is nowhere dense in $Y$.

Proof. The assertion is of local character about $Y$, and $Y^{\prime}$ is a dense open set of $Y$. So we may assume that $Y=Y(k)$ and there are $k$ meromorphic functions on $X$ such as in the above corollary. Hence $Y_{1}$ is nowhere dense in $Y$ by corollary of Theorem $\mathrm{I}$.

Lastly we discuss the case $Y=Y(m)$ (where $m$ is the dimension of fibers).

Lemma 4.2. Let $Z$ be a complex space and $W$ be a compact irreducible analytic subspace of $Z$ of dimension $m$, and $f_{1}, \cdots, f_{m}$ be meromorphic functions on $Z$ such that $f_{i} \| W$ is defined for any $i$ and $\left\{f_{1}\left\|W, \cdots, f_{m}\right\| W\right\}$ is independent. We put $F=f_{1} \times \cdots \times f_{m}, F_{0}$ $=F \| W, G=$ the graph of $F, G_{0}=$ the graph of $F_{c}$, and $G_{1}=G \mid W$ (the restriction of $G$ over $W$ ).

Now $\lambda$ be the natural projection of $G_{1}$ to $\boldsymbol{P}^{m}$ and $G_{1} \stackrel{\lambda_{1}}{\rightarrow} G_{1}^{\prime} \stackrel{\lambda_{2}}{\rightarrow} \mathbb{P}^{m}$ be the Stein factorization of $\lambda$. Then the holomorphic mapping $\lambda_{1} \mid G_{0}: G_{0} \rightarrow \lambda_{1}\left(G_{0}\right)$ is bimeromorphic and $\lambda_{1}\left(G_{0}\right)$ is an irreducible component of $G_{1}^{\prime}$. 
Proof. The graph $G_{0}$ of $F_{0}$ is an irreducible component of $G_{1}$. Let $G_{2}$ be the union of all the irreducible components of $G_{1}$ which are distinct from $G_{0}$. Since $\left\{f_{1}\left\|W, \cdots, f_{m}\right\| W\right\}$ is independent and $\operatorname{dim} W=m$, the proper holomorphic mapping $\lambda \mid G_{0}: G_{0} \rightarrow \mathbb{P}^{m}$ is surjective and of rank $m$. From this, it follows that $\lambda\left(G_{0} \cap G_{2}\right) \neq \boldsymbol{P}^{m}$, for $\lambda\left(G_{0} \cap G_{2}\right)=\boldsymbol{P}^{m}$ implies $G_{c} \subset G_{2}$. Hence our assertion is proved.

Theorem III. If the transcendence degree of the field $K_{t}^{\prime}$ is equal to the (complex) dimension of the fiber, then $K_{t}^{\prime}=K_{t}$.

Proof. By Lemma 4. 1, we may assume that $Y=Y(m)$ and that there are $m$ meromorphic functions $f_{1}, \cdots, f_{m}$ on $X$ such that $f_{i, t}$ is $\operatorname{defined}(i=1, \cdots, m)$ and the system $\left\{f_{1, t}, \cdots, f_{m, t}\right\}$ is independent. We put $F=f_{1} \times \cdots \times f_{m}$, and $G=$ the graph of $F \times \pi$ and $G_{t}=$ the graph of $F_{t}$, and denote the Stein factorization of $\hat{F \times \pi}$ by $G \rightarrow G^{\prime} \rightarrow \mathbb{P}^{m} \times Y$. Then, by Lemma $4.2, G_{t}$ is bimeromorphically equivalent to an irreducible component of $G^{\prime} \mid X_{t}$. Hence we can prove this theorem similarly to Theorem I.

\section{REFERENCES}

[1] Cartan, H., Quotients of complex analytic spaces, International Colloquium on Function Theory, Tata Inst. Fund. Res., Bombay, 1960.

[2] Grauert, G. and R. Remmert, Espaces analytiquement complets. C. R. Acad. Sci. Paris 245 (1956), 882-885.

[3] , Komplexe Räume, Math. Ann. 136 (1958), 245-318.

[4] Bilder und Urbilder analytischer Garben, Ann, of Math. 68 (1958), 393-443.

[5] Kuhlmann, N., Algebraic function fields on complex analytic spaces, Proc. Conf. on Complex Analysis, Minneapolis (1964), 155-172.

[6] Remmert, R., Meromorphe Functionen in compacten complexen Räumen, Math. Ann. 132 (1956), 277-288.

[7] Holomorphe und meromorphe Abbildungen komplexer Räume, Math. Ann. 133 (1957), 328-370.

[8] Stein, K., Maximale holomorphe und meromorphe Abbildungen, I, Amer. J. Math. 85 (1963), 298-315.

[9] Maximale holomorphe und meromorphe Abbildungen, II. Aemr. J. Math. 86 (1964), 823-868.

[10] Thimm, W., Untersuchungen über Deformationen, Math. Ann. 123 (1952), 19-31.

[11] , Über ausgeartete meromorphe Abbildungen, I, Math. Ann. 125 (1952), 145-164. 
